Gynäk. Rdsch. 1986;26:I-VI

\title{
Contents, Vol. 26, 1986
}

\section{No. 1 Originalarbeiten}

Reduktion der postoperativen Morbidität bei Kaiserschnitt durch risikoangepasste, perioperative, antibiotische Kurzzeitprophylaxe unter besonderer Berücksichtigung der

\section{Anaerobier}

Köppel, R.; Benz, J 1

In-vitro-Fertilisation und Embryotransfer an der UFK Basel

Pavic, N.; Birkhäuser, M.; Eppenberger, E.; Grund, M.; Neuenschwander, E

Endometrium-Zytologie mit Endoscan

Heinzl, S.; Hendry, M.; Gobat, S.; Zehnder, A 26

Kurzfassungen von Zeitschriftenartikeln

Plasminogenaktivatoren bei der Endometriose

Åstedt, B.; Nordenskjöld, F 35

B-HCG-Konzentrationen vor und nach dem Entstehen einer Präeklampsie

Said, M.E.; Campell, D.M.; Azzam, M.E.; MacGillivray, 136

Prämaligne Läsionen und mikroinvasives Karzinom der Zervix bei jüdischen Frauen: Eine epidemiologische Studie

Baram, A.; Galon, A.; Schachter, A 38

Plazentarer Widerstand und fetale Nabelschnurdurchströmung: Klinische Signifikanz

Trudinger, B.J.; Giles, W.B.; Cook, CM.; Bombardieri, J.; Collins, L 40

Uteroplazentare Durchblutung und Beurteilung der Wellenform in der Ultraschall-Dopplermethode im Vergleich von normaler und Risikoschwangerschaft

Trudinger, B.J.; Giles, W.B.; Cook, CM 42

Plasmasteroidhormone, Hormonrezeptoren und Thymidininkorporationsrate beim Endometriumkarzinom

Lindahl, B.; Aim, P.; Fernö, M.; Norgren, A.; Trope, C 43

Prophylaktische Insulinbehandlung des Gestationsdiabetes reduziert die Häufigkeit von

Makrosomie, operativer Geburtsbeendigung und Geburtstrauma

Coustan, D.; Imarah, J 45

Diagnose und Therapie des Ovarialkarzinoms: Eine aktuelle Übersicht

Barber, H.R.K 47

Prostacyklinproduktion der Plazenta bei normaler Schwangerschaft und EPH-Gestose

Walsh, S.W.; Behr, M.J.; Allen, N.H 49

Wirkung von Naloxon auf die Regulierung der hypophysären Gonadotropine und des Prolaktins durch endogene Opioide während des Menstruationszyklus

Snowden, E.U.; Khan-Dawood, F.S.; Dawood, M.Y 51

Pulsatile Gonadotropinsekretion während des menschlichen Menstruationszyklus: Nachweis der alternierenden Frequenz der Sekretion von gonadotropinfreisetzendem Hormon Reame, N.; Sauder, S.E.; Kelch, R.P.; Marshall, J.C 52

Induktion eines Progesteronrezeptors mit 17-ß-Östradiol beim menschlichen Ovarial- 


\section{karzinom}

Hamilton, T.C.; Behrens, B.C.; Louie, K.G.; Ozols, R.F 54

Zur Indikation und Methodik der Nephrosonographie und Isotopennephrographie in der

Schwangerschaft

Fischer, W.; Brunk, A.; Hawlitschek, R.; Lamm, D.; Prenzlau, P 56

Inhaltsverzeichnis III

Fortbildung

Das Amnioninfektionssyndrom aus der Sicht des Geburtshelfers

Salzer, $\mathrm{H} \quad 58$

Das Amnioninfektionssyndrom aus der Sicht des Neonatologen

Salzer, H.R 61

No. 2 Originalarbeiten

Behandlung der Mastopathie mit zyklischer Mastodynie: Klinische Ergebnisse und Hormonprofile

Kubista, E.; Müller, G.; Spona, J 65

Stérilet et nullipare en 1985

Kaufmann, E 80

Kurzfassungen von Zeitschriftenartikeln

Zelloberflächenveränderungen nach hochdosierter Progesteronbehandlung bei Endometriumkarzinom

Szarvas, Z.; Zsolnai, B 90

Sonographische Veränderungen zyklusabhängiger Endometriumveränderungen beim

Menschen

Sakamoto, C 91

Erhöhter Spiegel von Serum B-Endorphinen in einem Kollektiv von Frauen mit polycystischen Ovarien

Aleem, F.A.; Mclntosh, T 93

Ovulationsauslösung bei clomiphenrefraktären Patientinnen: Bedeutung des pulsatilen

GnRH in der klinischen Anwendung

Molloy, B.G.; Handcock, K.W.; Glass, M.R 94

Eine Gegenüberstellung von Prolaktin- und Steroidrezeptorbefunden sowie Histomorphologie des Mammakarzinoms

Hoffmann, G.; Schommer, M.; Schweikhart, G.; Grill, H.J 95

Auswirkung der Retinosäure auf humane Brustkrebszellen in vitro

Daxenbichler, G.; Marth, Ch.; Böck, G.; Dapunt, 097

Dramatischer Anstieg des plazentaren Proteins 5 in der Folge intravenöser Gaben kleinster

Dosen von Heparin

Menabawey, M.; Silman, R.; Riche, A.; Chard, T 98

Vergleich zwischen zytoplasmatischem Steroidrezeptor und Tumordifferenzierung und

Invasion von endometrialen Karzinomen

Quinn, M.A.; Pearce, P.; Fortune, D.W.; Koh, S.H.; Hsieh, C; Cauchi, M 99

Chorionbiopsie: klinische Erfahrungen, unmittelbare Komplikationen und Einstellung der

Patientinnen

Perry, T.B.; Vekemans, M.J.J.; Lippmann, A.; Hamilton, E.F.; Fournier, P.J.R. ... 102 Einsatz

von Interferon beim fortgeschrittenen Ovarialkarzinom

Abdulhay, G.; Di Saia, P.J.; Blessing, J.A.; Creasman, W.T 104 
Der Einfluss einer lokalen Applikation von Prostacyclin auf die plazentare Durchblutung beim Schaf

Landauer, M; Phernetton, T.M.; Parisi, V.M.; Clark, K.E.; Rankin, J.H.G 105

Der Einfluss von Dexamethason auf die Konzentration des sexualsteroidbindenden Proteins, des kortikosteroidbindenden Globulins und der Steroidhormone bei zyklierenden

Rhesus-Affen

Stanczyk, F.Z.; Petra, P.H.; Senner, J.W.; Novy, M.J 106

IV

Inhaltsverzeichnis

Exogen zugeführtes Prostacyclin verändert weder den plazentaren noch den fetalen Blutfluss bei Praeeklampsie

Jouppila, P.; Kirkinen, P.; Koivula, A.; Ylikorkala, 0

Einfluss des zytoplasmatischen Steroidrezeptorgehalts auf die Prognose des Endometrium-

karzinoms im Frühstadium

Creasman, W.T.; Soper, J.T.; McCarty, K.S., Jr.; McCarty, K.S., Sr.; Hinshaw, W.;

Clarke-Pearson, D.L 109

Prostaglandinkonzentration im Plasma schwangerer Frauen während Infusion von Angiotensin II

Strickland, D.M.;Cox, K.;McCubbin, J.H.;Whalley, PJ.;MacDonald, P.C.;Mitchell, M.D. 112

Effekt von Desogestrel-Ethinylöstradiol auf Hirsutismus, Androgene und sexualhormon-

bindendes Globulin bei Patientinnen mit polyzystischen Ovarien

Cullberg, G.; Hamberger, L.; Mattsson, L.-A.; Mobacken, H.; Samsioe, G 114

Fortbildung $\cdot$ Schwangerenvorsorge

Fortbildungstag der Österreichischen Gesellschaft für Gynäkologie und Geburtshilfe,

Bad Mitterndorf, 21. Juni 1984 Schwangerenvorsorge

Lechner, W.; Dapunt, $0 \quad 116$

Die Bedeutung von Risikofaktoren

Huber, J.C 119

Schwangerenvorsorge

Baumgarten, K 120

Betreuung der Risikogravidität anhand des Mutter-Kind-Passes

Burghardt, E 124

No. 3 Originalarbeiten

Über die Natur der Geburtsauslösung

Schneider, H.P.G.; Korte, K 129

Mammapalpation als Grundlage für eine frühere Diagnose maligner Mammaveränderungen

Sevelda, P.; Langer, M.; Skodler, W.D.; Salzer, H.; Kubista, E

Vaginalsmear und Hormonstatus

Gitsch, E.; Czerwenka, K.; Huber, J.; Spona, J 158

Kurzfassungen von Zeitschriftenartikeln

Vorgehen bei fetalem Hydrozephalus

Chervenak, F.A.; Berkowitz, R.L.; Tortora, M.; Hobbins, J.C 166

Resultate mit Beckenbestrahlung kombiniert mit Hydroxyharnstoff bei Patientinnen mit

Zervixkarzinom im Stadium lib mit negativem prätherapeutischem Lymphangiogramm

Piver, M.S.; Krishnamsetty, R.M.; Emrich, L.J 168 
Wirkung von Ritodrin auf den fetalen und den plazentaren Blutfluss sowie auf die Konzentration von Prostazyklin und Thromboxan

Jouppila, P.; Kirkinen, P.; Koivula, A.; Ylikorkala, 0

Flussgeschwindigkeitswellenformen im plazentaren Kreislauf der Mutter und im fetalen plazentaren Kreislauf

Trudinger, B.J.; Giles, W.B.; Cook, CM 171

Plazentarer Widerstand und fetale Nabelschnurdurchströmung: pathologische Korrelation

Giles, W.B.; Trudinger, B.J.; Baird, P.J 173

Inhaltsverzeichnis

$\mathrm{V}$

Ovulationsauslösung mittels pulsatiler GnRH-Behandlung bei Patientinnen mit polyzystischen Ovarien

Ory, S.J.; London, S.N.; Tyrey, L.; Hammond, C.B 174

Behandlung der Endometriose mit Danazol: Bericht über eine prospektive Studie von 6

Jahren

Buttram, V.C., Jr.; Reiter, R.C.; Ward, S 177

Zyklusabhängige Veränderungen bei Ziliarzellen sowie bei Zellhöhe und mitotischer

Aktivität des menschlichen Tubenepithels während der reproduktiven Phase

Donnez, J.; Casanas-Roux, F.; Caprasse, J.; Ferin, J.; Thomas, K 179

Cyproteronazetat als Initialtherapie und Dauerbehandlung beim Hirsutismus

Holdaway, I.M.; Croxson, M.S.; Ibbertson, H.K.; Sheehan, A.; Knox, B.; France, J. .181

Fortbildung · Die Sectio caesarea

Die Sectio caesarea - wechselnde Indikationen und Häufigkeit

Rudelstorfer, R 183

Beziehung zwischen perinataler Mortalität und Sectiofrequenz

Gruber, W 188

Auswirkungen des Entbindungsmodus auf das extrem unreife Kind in Beckenendlage

Dadak, C 190

No. 4 Originalarbeiten

Ergebnisse und Spätmorbidität nach der Inkontinenzoperation nach Marshall-Marchetti-

Burch zur Behandlung der Harnstressinkontinenz der Frau

Tinguely, J.-P.; Gaudenz, R 193

Entfernung eines okkulten intrauterinen Intrauterinpessarfragmentes unter hysterosko-

pischer Kontrolle

Karbowski, B.; Schneider, H.P.G 210

Kurzfassungen von Zeitschriftenartikeln

Die hämodynamische Überwachung bei der schweren Präeklampsie mit Lungenödem

Benedetti, T.J.; Kates, R.; Williams, V 215

EPH-Gestose: ein Ungleichgewicht zwischen plazentarer Prostacyclin- und Thromboxansynthese

Walsh, S.W 217

Der Zavanelli Handgriff: eine ungewöhnliche Methode zur Lösung von Problemen bei

Schulterdystokie

Sandberg, E.C 219

Ergebnisse der Second-Look-Operation nach initialer Chemotherapie bei Patientinnen mit malignen Ovarialtumoren 
Smirz, L.R.; Stemann, F.B.; Ulbright, T.N.; Sutton, G.P.; Ehrlich, C.G 220 Intraperitoneale Immuntherapie des epithelialen Ovarialkarzinoms mit Corynebacterium parvum

Berek, J.S.; Knapp, R.C.; Hacker, N.F.; Lichtenstein, A.; Jung, T.; Spina, C; Obrist, R.; Griffiths, C.T.; Berkowitz, R.S.; Parker, L.; Zighelboim, J.; Bast, R.C 222

Erfolgreiche Therapie von oligomenorrhoischen Sterilitätspatientinnen mit einer Kombination eines LHRH-Agonisten und exogen zugeführten Gonadotropinen

Fleming, R.; Haxton, M.J.; Hamilton, M.P.R.; McCune, G.S.; Black, W.P.; Macnaughton, M.C.; Coutts, J.R.T 225

VI

Inhaltsverzeichnis

Plasma Levonorgestrel und Progesteronspiegel bei Frauen, die mit Silastik bezogenen

Implantaten, die Levonorgestrel enthalten, behandelt wurden

Croxatto, H.B.; Diaz, S.; Brandeis, A.; Pavez, M.; Johansson, E.D.B 227

Suprapubische oder transurethrale Blasendrainage nach Kolposuspension bzw. Kolpor-

raphie

Andersen, J.T.; Heisterberg, L.; Hebjorn, S.; Petersen, K.; Stampe Sorensen, S.; Fischer-

Rasmussen, W.; Moisted Pedersen, L.; Nielsen, N.C229

Fortbildung

«Routineimpfungen» an der I. Universitäts-Frauenklinik. Rhesusprophylaxe und Röteln-

impfung im Wochenbett

Reichel, R 231

Impfungen in der Gravidität bei Tropenreisen

Ambrosch, F 235

Virusimpfungen in der Schwangerschaft

Hofmann, $\mathrm{H} 240$

Impfungen beim Neugeborenen

Salzer, H.R 243

Autorenregister

Suppl. 1 Cilag-Symposium über orale Kontrazeptiva

St. Moritz, 1984

Suppl. 2 Jahrestagung der Österreichische $\pi$ Gesellschaft für Gynäkologie und Geburtshilfe

S. Karger · Verlag für Medizin und Naturwissenschaften

Basel — München · Paris · London · New York · New Delhi — Singapore — Tokyo · Sydney

Dosierungsangaben von Medikamenten Alle Rechte vorbehalten.

Autoren und Verlag haben alle Anstrengungen unter- OhneschriftlicheGenehmigungdes

Verlagsdürfendiese

nommen, um sicherzustellen, dass Auswahl und Dosie- $\quad$ Publikation oder Teile daraus nicht

in andere Sprachen

rungsangaben von Medikamenten im vorliegenden Text übersetzt oder in irgendeiner Form

mit mechanischen

mit den aktuellen Vorschriften und der Praxis überein- oder elektronischen Mitteln

(einschliesslich Fotokopie,

stimmen. Trotzdem muss der Leserim Hinblick auf den Tonaufnahme und Mikrokopie)

reproduziert oder auf 
Stand der Forschung, Änderungen staatlicher Gesetzge- $\quad$ einem Datenträger oder einem Computersystem gespei-

bungen und den ununterbrochenen Fluss neuer For- chert werden.

schungsergebnissebezüglichMedikamentenwirkungund Nebenwirkungen darauf aufmerksam gemacht werden, dass unbedingt bei jedem Medikament der Packungs-prospekt konsultiert werden muss, um mögliche Änderungen im Hinblick auf Indikation und Dosis nicht zu übersehen. Gleiches gilt für spezielle Warnungen und Vorsichtsmassnahmen. Ganz besonders gilt dieser Hin-

weis für empfohlene neue und/oder nur selten gebrauchte (C) S. Karger AG, Postfach, CH- 4009 Basel (Sch weiz)

Wirkstoffe. Printed in Switzerland by Thür AG Offsetdruck, Pratteln 